\title{
A PARTICIPAÇÃO DE PRODUTORES RURAIS NAS COOPERATIVAS AGROPECUÁRIAS EM PAÍSES COM DIFERENTES NÍVEIS DE DESENVOLVIMENTO
}

\author{
FARMER PARTICIPATION IN AGRICULTURAL COOPERATIVES IN \\ COUNTRIES WITH DIFFERENT DEVELOPMENT LEVELS
}

\section{LA PARTICIPACIÓN DE PRODUCTORES RURALES EN COOPERATIVAS AGRÍCOLAS DE PAÍSES CON DIFERENTES NIVELES DE DESARROLLO}

\author{
Gabrieli dos Santos Amorim ${ }^{1}$ \\ https://orcid.org/0000-0002-3279-6993 \\ Adriano Lago ${ }^{2}$ \\ https://orcid.org/0000-0002-0499-102X \\ Mariele Boscardin ${ }^{3}$ \\ https://orcid.org/0000-0002-3308-4189 \\ Rosani Marisa Spanevello \\ https://orcid.org/0000-0002-4278-6895 \\ Camila Weber ${ }^{5}$ \\ https://orcid.org/0000-0003-0949-8960
}

Submissão: 12/11/2020 / Aceito: 11/05/2021 / Publicado: 04/02/2022.

\section{Resumo}

O objetivo desse estudo foi analisar os condicionantes da participação de produtores rurais nas cooperativas agropecuárias em países com diferentes níveis de desenvolvimento, enfatizando suas semelhanças e diferenças, indicando os possíveis motivos que levam a participação nas cooperativas agropecuárias e a predominância dos tipos de transações comerciais utilizando panoramas locais. Este trabalho foi elaborado por meio de uma revisão sistemática da literatura. Foram selecionadas publicações no período de 2013 a 2018 na base de dados SCOPUS ${ }^{\circledR}$. Os resultados demonstram que a participação de produtores rurais nas cooperativas agropecuárias é reforçada por características positivas e semelhantes entre os estudos, ao que se refere aos benefícios e vantagens como o aumento

\footnotetext{
${ }^{1}$ Mestre em Agronegócios pela Universidade Federal de Santa Maria (UFSM). E-mail: gabrieliamorim@hotmail.com

${ }^{2}$ Doutor em Agronegócios pela Universidade Federal do Rio Grande do Sul (UFRGS). Docente Professor Adjunto do Curso de Administração e do Programa de Pós-Graduação em Agronegócios da Universidade Federal de Santa Maria (UFSM), Campus Palmeira das Missões, Rio Grande do Sul, Brasil. E-mail: adrianolago@yahoo.com.br

${ }^{3}$ Doutoranda em Extensão Rural pela Universidade Federal de Santa Maria (UFSM) E-mail: marieleboscardin@hotmail.com

${ }^{4}$ Doutora em Desenvolvimento Rural pela Universidade Federal do Rio Grande do Sul (UFRGS). Docente Professora Adjunta do Curso de Zootecnia e do Programa de Pós-Graduação em Agronegócios da Universidade Federal de Santa Maria (UFSM), Campus Palmeira das Missões, Rio Grande do Sul, Brasil. Email: rspanevello@yahoo.com.br

5 Doutoranda em Agronegócios pela Universidade Federal do Rio Grande do Sul (UFRGS). E- mail: camyllaweber@gmail.com
} 
da renda, garantia de integração ao mercado e a eficiência técnica ou intensificação da agricultura, que se adequam às condições econômicas, demográficas e ambientais do local. Palavras-chave: Condicionantes da Participação. Cooperativismo. Revisão Sistemática.

\begin{abstract}
The aim of this study was to analyze the constraints of the farmer participation in agricultural cooperatives in countries with different levels of development, emphasizing their similarities and differences, indicating the possible reasons that lead the participation in agricultural cooperatives and the predominance of forms of transactions. using local panoramas. This paper was developed through a systematic literature review. Publications were selected from 2013 to 2018 in the SCOPUS® database. The results show that the participation of farmers in agricultural cooperatives is improved by positive and similar characteristics among the studies, regarding benefits and advantages such as increased income, guarantee of market integration and technical efficiency or intensification of agriculture, which suit the local economic, demographic and environmental conditions.
\end{abstract}

Keywords: Conditions of Participation. Cooperativism. Systematic Review.

\title{
Resumen
}

El objetivo de este estudio fue analizar las condiciones para la participación de productores rurales en cooperativas agrícolas en países con diferentes niveles de desarrollo, destacando sus similitudes y diferencias, señalando las posibles razones que conducen a la participación en cooperativas agrícolas y el predominio de tipos de transacciones. usando panoramas locales. Este trabajo se desarrolló mediante una revisión sistemática de la literatura. Las publicaciones de 2013 a 2018 fueron seleccionadas en la base de datos SCOPUS®. Los resultados demuestran que la participación de los productores rurales en las cooperativas agrarias se ve reforzada por características positivas y similares entre los estudios, en cuanto a los beneficios y ventajas como aumento de ingresos, garantía de integración al mercado y eficiencia técnica o intensificación de la agricultura, que se adaptan a las condiciones económicas, demográficas y medioambientales locales.

Palabras chave: Condiciones de participación. Cooperativismo. Revisión sistemática.

\section{INTRODUÇÃO}

O desenvolvimento pode ser definido como liberdade e aumento das capacidades humanas. Essa liberdade está relacionada à saúde, à educação, ao atendimento às necessidades materiais básicas e à participação na vida da comunidade. Já as tais capacidades estão associadas à realização dos objetivos políticos compartilhados pelas sociedades modernas (SEN, 1999). Além disso, o autor retrata que países em desenvolvimento necessitam de iniciativas das políticas públicas na criação de oportunidades sociais, a fim de contribuir diretamente para a expansão da capacidade humana e da qualidade de vida.

Essa abordagem permite reconhecer que a liberdade humana é o meio primordial do desenvolvimento e que as potencialidades individuais dependem fundamentalmente dos 
dispositivos econômicos, sociais e políticos. Na perspectiva de Bresser-Pereira (2014), salienta-se que o desenvolvimento econômico não pode ser injusto, ao abordar a mesma linha de pensamento de Sen (1999), baseada em desenvolvimento humano, em que o acesso à liberdade do indivíduo se realiza através da democracia.

Neste sentido, Nielsen (2013) destaca a importância de se basear em uma visão claramente articulada do que constitui o desenvolvimento, pois a literatura está repleta de terminologias concorrentes, como exemplos: países pobres, ricos, atrasados, avançados, subdesenvolvidos, desenvolvidos, em desenvolvimento, entre outros. As terminologias são fundamentadas por meio de indicadores sociais e econômicos que são oferecidos por órgãos mundiais.

Nos últimos anos, a pobreza, exclusão, desigualdades e conflitos persistem no mundo e interferem no aumento do Índice de Desenvolvimento Humano (IDH). Em 2018, por exemplo, índices e indicadores de desenvolvimento mostraram que as pessoas são formalmente mais educadas e têm mais acesso a mercadorias e serviços, mas a qualidade do desenvolvimento humano revela déficits (UNITED NATIONS DEVELOPMENT PROGRAMME-UNDP, 2018). Deste modo, o progresso não é linear ou garantido, e crises e desafios podem reverter ganhos, pois a persistente alta da desigualdade é uma dificuldade para sustentar o futuro e o progresso no desenvolvimento humano (UNDP, 2018).

O surgimento das cooperativas corrobora com a ideia de desenvolvimento humano. Lauschner (1993, p. 137) define cooperativa como "[...] organismo técnico, econômico e financeiro sob administração coletiva". Ele destaca a constituição social e econômica como finalidade de uma cooperativa. As cooperativas surgem de dificuldades do homem, como uma forma de preservar a força econômica e de vida dos indivíduos. São organizações de pessoas baseadas em princípios democráticos, com ênfase na questão social como requisito essencial para a formação de uma cooperativa (PINHO, 1982; SALES 2010).

As cooperativas são empresas centradas em pessoas de maneira democrática, controladas e dirigidas para que seus membros realizem suas necessidades e aspirações econômicas, sociais e culturais comuns (INTERNATIONAL CO-OPERATIVE ALLIANCE$I C A$, 2018). Chaddad e Cook (2004) corroboram essa perspectiva ao apontarem que cooperativa é uma organização que compreende um grupo de indivíduos que tem interesses em comum e acreditam que através de uma organização cooperativa, consigam alcançar seus interesses e objetivos mais facilmente. 
As cooperativas revelam uma preocupação a longo prazo com os aspectos sociais, econômicos e ambientais, baseado em ética, valores e princípios e com o bem-estar dos indivíduos. Nesta perspectiva, os valores elencados pelo cooperativismo são os elementos que diferenciam as cooperativas das demais organizações.

Conceitualmente, Teixeira et al. (2017) relatam que o cooperativismo no setor agropecuário está vinculado ao desenvolvimento do consumo e do comércio de produtos, que estimula o crescimento social, a preservação ambiental e evita o êxodo rural. Os autores consideram que as cooperativas agropecuárias têm importante influência nas propriedades rurais sobre os fatores econômicos e produtivos e também no âmbito social ao influenciar o homem do campo a permanecer no ambiente rural.

De modo geral, pode-se dizer que o cooperativismo se constitui em um modelo de negócio importante para amenizar, no cenário mundial atual, as instabilidades econômicas e sociais do segmento rural, especialmente de pequenos agricultores (TEIXEIRA et al., 2017). São eles que necessitam de serviços, que por sua vez, são escassos nas áreas rurais.

Neste caso, as cooperativas oferecem uma gama de atividades a estes agricultores, melhorando o alcance destes aos seguintes fatores: a gestão da propriedade, acesso ao mercado, obtenção de informação e conhecimento (FOOD AND AGRICULTURE ORGANIZATION OF THE UNITED NATIONS - FAO, 2012).

Além disso, elas podem apoiar e fortalecer o atendimento da crescente demanda por alimentos nos mercados locais, nacionais e internacionais (FAO, 2012). Para os agricultores, a participação em cooperativas favorece e contribui com o desenvolvimento de estratégias e fortalecimento da tomada de decisão de fatores importantes, independente de qual é a sua localização no mundo (FAO, 2018a).

A nível global, regiões ou países se classificam de acordo com o seu padrão de desenvolvimento social e econômico, conforme se detalha na metodologia deste estudo. Neste caso, independente da classificação do país (desenvolvidos, em desenvolvimento ou subdesenvolvidos), as cooperativas estão presentes. Em especial, para os países subdesenvolvidos e em desenvolvimento, a Organização Internacional do Trabalho (OIT) divulgou o documento de Recomendação $n^{\circ} 193$ para que os governos destas nações adotem políticas de expansão do cooperativismo, devido à contribuição das cooperativas para o desenvolvimento social, econômico e sustentável da população (OIT, 2012). 
Neste estudo, objetivou-se analisar os condicionantes da participação de produtores rurais nas cooperativas agropecuárias em países com diferentes níveis de desenvolvimento em função desta importância dada ao cooperativismo como uma organização da sociedade civil, que tem como um de seus objetivos assegurar os interesses de seus associados. Com o intuito de demonstrar as semelhanças e diferenças dos condicionantes em diferentes localidades. Para a elaboração desta pesquisa foi utilizada a revisão sistemática da literatura entre os anos de 2013-2018 mediante a base de dados bibliográfica SCOPUS ${ }^{\circledR}$.

Além disso, a relevância da temática é referente à perspectiva de que os resultados possam servir de apoio para o desenvolvimento de estratégias e tomada de decisão em relação à participação de produtores rurais em cooperativas agropecuárias. Esse é um fator importante de apoio aos produtores agrícolas e pecuários independente de qual a sua localização no mundo.

\section{PERCURSO METODOLÓGICO}

O estudo está baseado em uma revisão sistemática da literatura. A metassíntese qualitativa é utilizada como modalidade de pesquisa para a compreensão e análise de conteúdo dos estudos selecionados.

Alencar e Almouloud (2017) consideram a metassíntese qualitativa como modalidade de pesquisa para revisões sistemáticas que tenham como princípio uma abordagem qualitativa, direcionada para compreender estudos primários, operando por meio de comparações de semelhanças e diferenças entre as pesquisas. A metassíntese qualitativa integra resultados qualitativos de pesquisas em forma de síntese, descrevendo-os de forma integrada e interpretativa. Primeiro de forma individual para depois os alinhavar entre os estudos. Não se considera a significância estatística, mas as particularidades e interpretações dos estudos (LOPES; FRACOLLI, 2008).

Ainda, o estudo se classifica em uma revisão sistemática, utilizando um tema como fonte de dados na literatura e procurando reunir todo o conteúdo disponível sobre o assunto. Investiga-se o tema escolhido por meio de métodos sistematizados e abrangentes de busca e viabiliza-se ao incorporar um resumo dos estudos de determinada temática de forma relevante e crítica (SAMPAIO; MANCINI, 2007; LOPES; FRACOLLI, 2008).

Utilizou-se um levantamento de dados da base SCOPUS ${ }^{\circledR}$ pois é uma base mundial e de atualização diária. $\mathrm{O}$ acesso à base de dados foi realizado via sistema Comunidade 
Acadêmica Federada (CAPES/Brasil). As etapas realizadas durante o processo de seleção dos documentos são apresentadas no Quadro 1.

Quadro 1: Critérios de seleção dos documentos na base de dados Scopus

\begin{tabular}{|c|c|c|}
\hline \multicolumn{2}{|c|}{ CRITÉRIOS DE SELEÇÃO DE DOCUMENTOS: SCOPUS } & $\begin{array}{l}\text { TOTAL DE } \\
\text { ARTIGOS }\end{array}$ \\
\hline $\begin{array}{l}\text { Termos de busca encontrados no título do } \\
\text { artigo, resumo e palavras-chave. }\end{array}$ & $\begin{array}{c}\text { “agricultural cooperatives" } \\
\text { ou } \\
\text { “cooperativismo agricultural" } \\
\text { ou } \\
\text { "agribusiness cooperatives" }\end{array}$ & 1.071 \\
\hline Ano de publicação & $2013-2018$ & 437 \\
\hline Área das publicações & $\begin{array}{l}\text { Ciências Sociais } \\
\text { ou } \\
\text { Ciências Agrárias }\end{array}$ & 318 \\
\hline Tipo de documento & Artigo & 264 \\
\hline Tipo da fonte & Revistas & 263 \\
\hline Palavras-Chave & $\begin{array}{c}\text { "agricultural cooperative" } \\
\text { ou } \\
\text { "agricultural cooperatives" }\end{array}$ & 121 \\
\hline Palavras-Chave no título & $\begin{array}{c}\text { "cooperatives" } \\
\text { ou } \\
\text { "cooperativism" }\end{array}$ & 71 \\
\hline \multicolumn{2}{|c|}{ TOTAL DE DOCUMENTOS SELECIONADOS: } & 71 \\
\hline \multicolumn{2}{|c|}{$\begin{array}{l}\text { Leitura dos resumos dos artigos: } \\
\text { seleção dos artigos que se assemelham ao objetivo do trabalho. }\end{array}$} & 36 \\
\hline \multicolumn{2}{|c|}{$\begin{array}{l}\text { Leitura dos artigos: artigos que não abordam } \\
\text { os objetivos gerais do trabalho serão excluídos da amostra. }\end{array}$} & 12 \\
\hline \multicolumn{2}{|c|}{ TOTAL FINAL DE DOCUMENTOS SELECIONADOS: } & 12 \\
\hline
\end{tabular}

Fonte: Elaborado pelos autores (2018).

Em relação ao total de documentos selecionados que resultaram em 71 artigos, realizou-se a leitura dos resumos de cada artigo e selecionaram-se apenas os artigos que apresentavam objetivos alinhados à temática pesquisada. Deste modo, foram escolhidos 36 artigos, a partir deles foi realizada a leitura na integra dos documentos e selecionados 12 artigos que demonstravam similaridade. Através dos 12 artigos escolhidos, realizou-se a revisão sistemática por meio da metassíntese qualitativa dos estudos.

Nessa perspectiva, além dos resultados encontrados em cada estudo individualmente, este trabalho associa um panorama da situação dos países onde foram realizadas tais pesquisas. Além disso, foi utilizada a classificação das pesquisas por nível de desenvolvimento dos países. O objetivo foi destacar as possíveis diferenças nos resultados da participação dos produtores rurais nas cooperativas agropecuárias em países com 
diferentes níveis de desenvolvimento. Deste modo, é necessário compreender como se dá a classificação desses países.

Neste contexto, existem indicadores mundiais que classificam o desenvolvimento social e econômico dos países, no intuito de medir e categorizar, podendo-se destacar o Índice de Desenvolvimento Humano (IDH) e o coeficiente de Gini. Este último se refere à medida do desvio da distribuição de renda entre indivíduos ou famílias dentro de um país a partir de uma distribuição perfeitamente igual, sendo que um valor de 0 representa igualdade absoluta e um valor de 1 desigualdade absoluta (UNDP, 2018).

O IDH é medido de acordo com três dimensões: a renda para um melhor padrão de vida, a educação que desenvolve o conhecimento dos indivíduos e a saúde para uma vida longa e saudável. O IDH varia entre 0 e 1, ou seja, quanto mais se aproxima de 1 maior é o desenvolvimento do país e tem como objetivo avaliar outros aspectos além dos econômicos, como os sociais. Assim, amplia a perspectiva de desenvolvimento humano, não só pelo que a renda pode gerar, mas sim pelas capacidades e oportunidades dos indivíduos, onde ela é considerada apenas como um meio de desenvolvimento e não como um fim (UNDP, 2018).

Nesta perspectiva, diversos órgãos, como Fundo Monetário Internacional, Programa das Nações Unidas para o Desenvolvimento, Banco Mundial e a Organização Mundial do Comércio, abordam a questão das taxonomias de desenvolvimento para os países do mundo por meio de indicadores de desenvolvimento (NIELSEN, 2013).

Diante do exposto, utilizando-se da classificação dada pelo Programa das Nações Unidas para o Desenvolvimento, através de seus indicadores IDH e Coeficiente de Gini, os países dos estudos selecionados para a revisão sistemática foram classificados de acordo com o seu nível de desenvolvimento, que pode ser visualizado no Quadro 2.

Quadro 2: Classificação por nível de desenvolvimento dos países apresentados nos estudos.

\begin{tabular}{|c|c|c|}
\hline Nível de desenvolvimento & Continente & Países \\
\hline Desenvolvido & Europeu & Finlândia \\
\hline Em desenvolvimento & Asiático & China e Índia \\
\hline Subdesenvolvido & Africano & Etiópia e Ruanda \\
\hline
\end{tabular}

Fonte: Elaborado pelos autores (2018). 
A classificação de desenvolvimento pelos três blocos foi utilizada como a principal variável para indicar as semelhanças e diferenças entre os resultados relevantes que as pesquisas apresentam, de acordo com o nível de desenvolvimento de cada região, em relação à participação de produtores rurais em cooperativas agropecuárias. Destaca-se que a classificação por nível de desenvolvimento não foi parâmetro da seleção de artigos na base de dados. Os países foram considerados aleatoriamente na seleção, a partir das palavras chave e do alinhamento dos artigos aos objetivos do estudo.

\section{PANORAMA DOS ESTUDOS SELECIONADOS}

A revisão sistemática da literatura contempla um panorama das características de amostras de produtores rurais dos estudos selecionados. Pode ser evidenciado um aspecto em comum sobre o índice de escolaridade dos produtores. Os autores Verhofstadt e Maerstens $(2014,2015)$ indicam que um ano adicional de educação do chefe do agregado familiar aumenta a probabilidade de adesão à cooperativa em 2,2\% e que a média de escolaridade dos produtores são entre quatro a cinco anos de estudos.

Na perspectiva de Mojo, Fischer e Degefa (2017), a média de escolaridade dos produtores são entre dois a quatro anos de estudos e os membros e não-membros são semelhantes em seu nível de educação. Já produtores rurais com maior nível de escolaridade têm cerca de 7,8\% maior probabilidade de participação em uma cooperativa, dado este que se reafirma no estudo anterior dos autores Mojo, Fischer e Degefa (2015). Entretanto, os autores Chagwiza, Muradian e Ruben (2016) salientam que famílias membros de cooperativas que são chefiados por pessoas idosas, possuem um nível de educação superior, em comparação com os não membros.

Para Ahmed e Mesfin (2017), um total de 64\% dos produtores rurais da amostra não obtiveram educação formal e a filiação na cooperativa é mais eficaz para produtores com maior nível educacional. Ainda, Kumar et al., (2018) relatam que a média de escolaridade dos produtores são entre cinco a sete anos de estudos. Segundo os autores Ma e Abdulai (2016, 2017), o número médio de anos em escolaridade é de 7,6. Entretanto, os autores Ma, Abdulai e Goetz (2018) ainda retratam que o aumento da educação torna maior a probabilidade de escolha para ser um membro cooperativo, já que a baixa probabilidade de 
escolha para ser um membro ocorre com produtores com menos de nove anos de escolaridade.

Deste modo, identifica-se que as escolaridades dos produtores divergem de acordo com o nível de desenvolvimento dos países, visto que para países subdesenvolvidos a média de escolaridade é menor em relação aos países em desenvolvimento. $\mathrm{O}$ índice de educação é um dado que complementa esta diferenciação evidenciada anteriormente no nível de escolaridade dos produtores rurais da amostra. O índice de educação é uma média de anos de escolaridade de adultos e anos de escolaridade esperados de crianças com amplitude de 0 a 1 (UNDP, 2018).

O índice de educação dos países em estudo é respectivamente: Finlândia 0,905, China 0,644, Índia 0,556, Ruanda 0,450 e Etiópia 0,327 (UNDP, 2018). Demonstra-se que o país com maior nível de educação é o desenvolvido, seguido dos países em desenvolvimento e dos subdesenvolvidos. No entanto, estes dados contemplam a ideia que o índice de educação se mostra de forma decrescente em relação ao nível de desenvolvimento dos países e corrobora com a caracterização da escolaridade da amostra de produtores rurais dos estudos.

A probabilidade de aderir às cooperativas agropecuárias entre os produtores da amostra, na maioria dos estudos, é maior em produtores com as famílias de maior tamanho, mais posse de terras e localização mais próxima à cooperativa (MA; ABDULAI 2016; KUMAR et al., 2018; VERHOFSTADT; MAERSTENS, 2014, 2015; ABATE; FRANCESCONI; GETNET, 2014; CHAGWIZA; MURADIAN; RUBEN, 2016; MOJO; FISCHER; DEGEFA, 2017; AHMED; MESFIN, 2017). Neste caso, o nível de desenvolvimento dos países em estudo não interfere nas características para a adesão das cooperativas agropecuárias, prevalecendo os mesmos atributos tanto para países em desenvolvimento quanto aos subdesenvolvidos.

Portanto, a não diferenciação nos quesitos prováveis para adesão da cooperativa por produtores rurais entre os países pode estar ligada ao intuito dos mesmos na busca pela satisfação das suas necessidades econômicas e sociais. Segundo Teixeira et al. (2017), as cooperativas atuam com um propósito democrático e organizado na busca de satisfazer as necessidades econômicas e sociais dos indivíduos.

De acordo com os estudos, totaliza-se um número relevante de produtores rurais, vistos no Quadro 3. São 5.933 produtores que abrangem diferenciações entre o número de 
membros e não-membros das cooperativas agropecuárias. Fato este essencial para indicar as diferenças que a participação cooperativa estabelece entre os cooperados e não cooperados.

Quadro 3: Panorama das amostras de produtores rurais dos estudos selecionados.

\begin{tabular}{|c|c|c|}
\hline $\begin{array}{c}\text { NÍVEL DE } \\
\text { DESENVOLVIMENTO }\end{array}$ & AUTORES & PANORAMA \\
\hline \multirow{7}{*}{ PAÍSES SUBDESENVOLVIDOS } & $\begin{array}{l}\text { Abate, Francesconi e Genet } \\
\text { (2014) }\end{array}$ & $\begin{array}{c}1.638 \text { produtores } \\
557 \text { membros } \\
\text { 1.081 não membros }\end{array}$ \\
\hline & $\begin{array}{l}\text { Chagwiza, Muradian e Ruben } \\
\text { (2016) }\end{array}$ & $\begin{array}{c}384 \text { produtores } \\
192 \text { membros } \\
192 \text { não membros }\end{array}$ \\
\hline & Mojo, Fischer e Degefa (2017) & $\begin{array}{c}305 \text { produtores } \\
139 \text { membros } \\
166 \text { não membros } \\
\end{array}$ \\
\hline & Mojo, Fischer e Degefa (2015) & $\begin{array}{c}305 \text { produtores } \\
139 \text { membros } \\
166 \text { não membros }\end{array}$ \\
\hline & Ahmed e Mesfin (2017) & $\begin{array}{c}250 \text { produtores } \\
121 \text { membros } \\
129 \text { não membros } \\
\end{array}$ \\
\hline & Verhofstadt e Maerstens (2014) & $\begin{array}{c}389 \text { produtores } \\
251 \text { membros } \\
138 \text { não membros }\end{array}$ \\
\hline & Verhofstadt e Maerstens (2015) & $\begin{array}{c}389 \text { produtores } \\
154 \text { membros } \\
235 \text { não membros } \\
\end{array}$ \\
\hline \multirow{4}{*}{$\begin{array}{c}\text { PAÍSES EM } \\
\text { DESENVOLVIMENTO }\end{array}$} & Ma e Abdulai (2016) & 481 produtores \\
\hline & Ma e Abdulai (2017) & $\begin{array}{c}481 \text { produtores } \\
208 \text { membros } \\
273 \text { não membros }\end{array}$ \\
\hline & Ma, Abdulai e Goetz (2018) & $\begin{array}{c}481 \text { produtores } \\
208 \text { membros } \\
273 \text { não membros } \\
\end{array}$ \\
\hline & Kumar et al. (2018) & $\begin{array}{l}148 \text { produtores } \\
60 \text { membros } \\
88 \text { não membros }\end{array}$ \\
\hline PAÍS DESENVOLVIDO & Alho (2015) & $\begin{array}{l}682 \text { produtores } \\
276 \text { produtores de carne } \\
406 \text { produtores de leite }\end{array}$ \\
\hline
\end{tabular}

Fonte: Elaborado pelos autores (2018).

\section{DESENVOLVIMENTO AGRÍCOLA DOS PAÍSES ANALISADOS}

Cada país pertencente ao seu nível de desenvolvimento possui características próprias no que diz respeito, principalmente, às cooperativas agropecuárias e aos seus produtos comercializados, os quais se adequam às condições econômicas, demográficas e 
ambientais do local. Segundo Lima et al. (2019), as propriedades rurais podem possuir semelhanças históricas e culturais, mas cada uma pode diferir em seus recursos físicos, biológicos e humanos. Deste modo, torna-se significativo identificar os resultados predominantes dos estudos entre os níveis de desenvolvimento dos países. A seguir está o Quadro 4, o qual indica a relação dos estudos publicados referente aos seus objetivos e resultados por nível de desenvolvimento dos países.

Quadro 4: Síntese dos objetivos e resultados dos estudos selecionados.

\begin{tabular}{|c|c|c|}
\hline $\begin{array}{c}\text { NÍVEL DE } \\
\text { DESENVOLVIMENTO/ } \\
\text { AUTORES } \\
\end{array}$ & OBJETIVO & RESULTADOS \\
\hline $\begin{array}{l}\text { Subdesenvolvido } \\
\text { Abate, Francesconi e Getnet } \\
\text { (2014) }\end{array}$ & $\begin{array}{l}\text { Analisar o impacto das cooperativas } \\
\text { agropecuárias na eficiência técnica de } \\
\text { pequenos produtores rurais. }\end{array}$ & $\begin{array}{l}\text { Aumento da eficiência técnica dos } \\
\text { produtores rurais, acesso aos } \\
\text { insumos, treinamentos, serviços e } \\
\text { informações. }\end{array}$ \\
\hline $\begin{array}{c}\text { Subdesenvolvido } \\
\text { Chagwiza, Muradian e Ruben } \\
\text { (2016) }\end{array}$ & $\begin{array}{l}\text { Analisar as vantagens da participação } \\
\text { em cooperativas agropecuárias na } \\
\text { integração de mercado e no } \\
\text { desenvolvimento econômico dos } \\
\text { pequenos produtores de leite. }\end{array}$ & $\begin{array}{l}\text { Aumento da renda leiteira, adesão } \\
\text { a inovações tecnológicas e } \\
\text { integração ao mercado confiável. }\end{array}$ \\
\hline $\begin{array}{c}\text { Subdesenvolvido } \\
\text { Mojo, Fischer e Degefa (2017) }\end{array}$ & $\begin{array}{l}\text { Analisar os determinantes e os } \\
\text { impactos econômicos da participação } \\
\text { de produtores de café em cooperativas } \\
\text { agropecuárias. }\end{array}$ & $\begin{array}{l}\text { Aumento do desempenho } \\
\text { econômico (renda) e estabilização } \\
\text { de preço do produto. }\end{array}$ \\
\hline $\begin{array}{c}\text { Subdesenvolvido } \\
\text { Mojo, Fischer e Degefa (2015) }\end{array}$ & $\begin{array}{l}\text { Analisar o impacto da participação em } \\
\text { cooperativas agropecuárias } \\
\text { desempenho social e ambiental de } \\
\text { produtores de café. }\end{array}$ & $\begin{array}{l}\text { Intensificação do capital humano } \\
\text { e social e baixo desempenho } \\
\text { ambiental dos produtores. }\end{array}$ \\
\hline $\begin{array}{c}\text { Subdesenvolvido } \\
\text { Ahmed e Mesfin (2017) }\end{array}$ & $\begin{array}{l}\text { Analisar o impacto da participação em } \\
\text { cooperativas agropecuárias no bem- } \\
\text { estar de pequenos agricultores. }\end{array}$ & $\begin{array}{l}\text { Impacto positivo no bem-estar da } \\
\text { comunidade rural. }\end{array}$ \\
\hline $\begin{array}{l}\text { Subdesenvolvido } \\
\text { Verhofstadt e Maerstens } \\
\text { (2014) }\end{array}$ & $\begin{array}{l}\text { Analisar as vantagens da participação } \\
\text { de produtores rurais em cooperativas } \\
\text { agropecuárias de milho e horticultura } \\
\text { no desempenho agrícola. }\end{array}$ & $\begin{array}{l}\text { Aumento do desempenho agrícola } \\
\text { (renda) e intensificação da } \\
\text { agricultura, utilização de insumos, } \\
\text { resultados mais significativos } \\
\text { para a cooperativa de milho. }\end{array}$ \\
\hline $\begin{array}{c}\text { Subdesenvolvido } \\
\text { Verhofstadt e Maerstens } \\
(2015) \\
\end{array}$ & $\begin{array}{l}\text { Analisar a inclusão e a eficácia das } \\
\text { cooperativas agropecuárias na pobreza } \\
\text { rural dos produtores. }\end{array}$ & $\begin{array}{l}\text { Elevação de renda e redução da } \\
\text { pobreza. }\end{array}$ \\
\hline $\begin{array}{l}\text { Em desenvolvimento } \\
\text { Ma e Abdulai (2016) }\end{array}$ & $\begin{array}{l}\text { Analisar as vantagens da participação } \\
\text { dos produtores rurais em cooperativas } \\
\text { agrícolas de maçã em indicadores de } \\
\text { produção, retornos líquidos e renda } \\
\text { familiar. }\end{array}$ & $\begin{array}{l}\text { Aumento no desempenho em } \\
\text { indicadores de produção, retornos } \\
\text { líquidos e renda familiar. }\end{array}$ \\
\hline $\begin{array}{l}\text { Em desenvolvimento } \\
\text { Ma e Abdulai (2017) }\end{array}$ & $\begin{array}{l}\text { Analisar as vantagens da participação } \\
\text { de produtores rurais em cooperativas } \\
\text { agrícolas de maçã, no preço de } \\
\text { produção, renda bruta, lucro agrícola e } \\
\text { ROI. }\end{array}$ & $\begin{array}{l}\text { Aumento no preço de produção, } \\
\text { renda bruta, lucro agrícola e ROI. }\end{array}$ \\
\hline $\begin{array}{c}\text { Em desenvolvimento } \\
\text { Ma, Abdulai e Goetz (2018) }\end{array}$ & $\begin{array}{l}\text { Analisar as vantagens da participação } \\
\text { de produtores rurais em cooperativas }\end{array}$ & $\begin{array}{l}\text { Cooperados têm maior propensão } \\
\text { a utilizar adubos orgânicos em }\end{array}$ \\
\hline
\end{tabular}




\begin{tabular}{|c|c|c|}
\hline & $\begin{array}{l}\text { agrícolas de maçã no investimento em } \\
\text { adubação orgânica e fertilizantes } \\
\text { químicos. }\end{array}$ & relação aos fertilizantes químicos. \\
\hline $\begin{array}{l}\text { Em desenvolvimento } \\
\text { Kumar et al. (2018) }\end{array}$ & $\begin{array}{l}\text { Analisar o papel das cooperativas de } \\
\text { laticínios no desenvolvimento agrícola } \\
\text { dos produtores, na produção de leite, } \\
\text { retornos líquidos por litro e as medidas } \\
\text { de segurança alimentar. }\end{array}$ & $\begin{array}{l}\text { Aumento no desempenho agrícola } \\
\text { na produção de leite, retornos } \\
\text { líquidos por litro e na } \\
\text { conformidade com as medidas de } \\
\text { segurança alimentar. }\end{array}$ \\
\hline $\begin{array}{l}\text { Desenvolvido } \\
\text { Alho (2015) }\end{array}$ & $\begin{array}{l}\text { Analisar a heterogeneidade das } \\
\text { estruturas das cooperativas } \\
\text { agropecuárias ao abordar a questão de } \\
\text { como entregar benefícios aos } \\
\text { produtores rurais de leite e de carne. }\end{array}$ & $\begin{array}{l}\text { Heterogeneidade organizacional, } \\
\text { segurança de mercado, preço } \\
\text { competitivo e benefícios } \\
\text { relacionados à participação em } \\
\text { controle e tomada de decisões. }\end{array}$ \\
\hline
\end{tabular}

Fonte: Elaborado pelos autores (2018).

Em referência ao Quadro 4, nota-se que, no grupo de países subdesenvolvidos, a participação cooperativa garante diversos benefícios como o aumento de renda agropecuária dos produtores rurais e a eficiência técnica ou intensificação da agricultura. Assim, promove a produtividade dos locais em estudo, resultando na redução da pobreza e o aumento do bem-estar das comunidades rurais.

Nos países em desenvolvimento, as principais vantagens da participação são referentes ao aumento da renda em sua maioria, produtividade e preço para os produtores rurais. Para o país desenvolvido, o principal destaque do estudo é a segurança de mercado e o preço competitivo dado pela participação cooperativa.

De modo geral, o principal destaque dado à participação em cooperativas agropecuárias na maior parte dos estudos, refere-se ao aumento da renda agrícola. Ela garante ao produtor diversos benefícios que muitas vezes se sucedem em outros, os quais podem estar vinculados com o acesso ao crédito, à intensificação da produção e à garantia de mercado, onde o produtor possa acessar os insumos, garantir um nível de produção e ter a quem comercializar o seu produto. Fato que eleva o seu desempenho agrícola e garante o bem-estar das propriedades rurais.

Desta maneira, é relevante entender a situação dos países ao caracterizar o seu desenvolvimento agrícola. Nos países subdesenvolvidos, por exemplo, é possível analisar a aparição de resultados como a redução da pobreza e o bem-estar da comunidade rural.

A população da Etiópia é de 104,957 milhões de habitantes com uma área agrícola de 36.259 (x1.000 ha) e a população de Ruanda é de 12,208 milhões de habitantes com uma área agrícola de 1.809,5 (x1.000 ha) (BANCO MUNDIAL, 2018; FAO, 2018b). As 
cooperativas na Etiópia, antes de 1990, não representavam em sua estrutura os princípios cooperativos.

A partir do final da década de 1990, o governo da Etiópia tomou a iniciativa e interesse pelas cooperativas e as mesmas passaram a fazer parte do desenvolvimento rural do país. Diante desse fato, foi possível alcançar um nível maior de integração de mercado para os pequenos produtores rurais, alavancando a renda. A produção de leite por pequenos produtores da Etiópia é um exemplo (ABATE; FRANCESCONI; GETNET, 2014). O setor agrícola em Ruanda é relevante para o país, sendo que as cooperativas agropecuárias possuem rápida disseminação e são mais prevalentes nos subsetores de horticultura, café e milho e são principalmente iniciadas pelo governo do país (VERHOFSTADT; MAERSTENS, 2014).

Já nos países em desenvolvimento, a produtividade é o principal resultado visto nos estudos da China e da Índia. A população da China é de 1,386 bilhões de habitantes com uma área agrícola de 515.357,7 (x1.000 ha) e a população da Índia é de 1,339 bilhões de habitantes com uma área agrícola de 179.721 (x1.000 ha) (BANCO MUNDIAL, 2018; FAO, 2018b).

Na China, as províncias de Gansu, Shaanxi e Shandong cobrem mais da metade do total de pomares de maçãs do país. O governo Chinês apoia e facilita a criação de cooperativas de forma financeira e política. Além disso, a produção de maçãs é voltada principalmente para comercialização e para garantia de subsistência dos produtores (MA; ABDULAI, 2016; MA; ABDULAI, 2017; MA; ABDULAI; GOETZ, 2018).

No caso da Índia, a Revolução Branca iniciou em 1970 em pequenas propriedades leiteiras. Foi bem-sucedida nas áreas de criação de gado e trouxe muitos benefícios. Porém, ainda há algumas mudanças para serem realizadas, pois essa Revolução aumentou a produtividade do leite, mas não transformou o sistema agrícola e muitos dos pequenos produtores ainda utilizam práticas tradicionais (DEKA et al., 2015).

As cooperativas e empresas privadas estabelecidas próximas às aldeias compram leite de produtores indianos. Elas revisam os preços da compra de leite periodicamente baseados na oferta e na demanda. Os preços fornecidos pelas cooperativas, apoiadas pelo governo, são os mesmos que empresas de laticínios privadas seguem como referência (UNITED STATES DEPARTMENT OF AGRICULTURE-USDA, 2017). 
No país desenvolvido, a segurança de mercado e o preço competitivo são os principais resultados do estudo. A população da Finlândia é de 5,511 milhões de habitantes com uma área agrícola de 2.267,1 (x1.000 ha) (BANCO MUNDIAL, 2018; FAO, 2018b). Na Finlândia, em terrenos montanhosos, a pecuária é uma atividade econômica vital e existem poucas alternativas a não ser a criação de animais. Baseada na agricultura familiar, essa produção traz diversas oportunidades que incrementam suas terras com atividades de turismo e silvicultura, dado perfil geográfico do país com diversas florestas e lagos e o clima frio (FAO, 2018b).

$\mathrm{Na}$ União Europeia, mais de $40 \%$ das commodities agropecuárias são comercializadas por cooperativas, enquanto na África apenas 5\%. Essa diferença se deve ao fato de os produtores do continente africano venderem sua produção indiretamente na busca por transações rápidas e não pela falta de cooperativas para comercializar sua produção. Essa situação apenas prejudica a integração de cooperativas africanas nas cadeias de valor em relação aos países desenvolvidos (FRANCESCONI, 2017).

\section{PRINCIPAIS RESULTADOS DOS ESTUDOS ENTRE OS NÍVEIS DE DESENVOLVIMENTO DOS PAÍSES}

É necessário categorizar e descrever os principais resultados obtidos entre os estudos para detalhar as semelhanças e diferenças entre eles, dado o nível de desenvolvimento e as características dos países apontados na seção anterior.

Os principais resultados revelam duas situações. Na primeira, há mais de um resultado em evidência para cada país e, na segunda, os mesmos resultados aparecem em países com diferentes níveis de desenvolvimento. A partir destas manifestações, é possível elencar considerações pertinentes às semelhanças ao que se refere às vantagens e aos benefícios desses resultados em relação aos produtores rurais membros de cooperativas. Isso, independentemente do nível de desenvolvimento dos países e as diferenças que expõe as características que são mais predominantes entre os resultados, dado o nível de desenvolvimento dos países.

No Quadro 5, está apresentado o agrupamento dos principais resultados de acordo com o nível de desenvolvimento do país e o número de publicações em que são citados. 
Quadro 5: Agrupamento dos principais resultados, semelhantes, dos estudos por número de aparecimento nas publicações entre os níveis de desenvolvimento

\begin{tabular}{|c|c|c|}
\hline PRINCIPAIS RESULTADOS & NÍVEL DE DESENVOLVIMENTO & $\mathbf{N}^{\mathbf{0}}$ DE PUBLICAÇÕ ES \\
\hline $\begin{array}{c}\text { Eficiência técnica (acesso a insumos, } \\
\text { tecnologia, treinamento e serviços) }\end{array}$ & Subdesenvolvido & 3 \\
\hline Renda & $\begin{array}{c}\text { Subdesenvolvido e em } \\
\text { desenvolvimento }\end{array}$ & 3 \\
\hline Preço & $\begin{array}{c}\text { Subdesenvolvido, em desenvolvimento } \\
\text { e desenvolvido }\end{array}$ & 2 \\
\hline Produtividade & Em desenvolvimento & 3 \\
\hline Retorno de investimento & Em desenvolvimento & 2 \\
\hline Integração de mercado & Desenvolvido e Subdesenvolvido & 1 \\
\hline Bem-estar & Subdesenvolvido & 1 \\
\hline Redução de pobreza & Subdesenvolvido & 1 \\
\hline Capital humano, social e ambiental & Subdesenvolvido & 1 \\
\hline Adubação orgânica & Em desenvolvimento & 1 \\
\hline Controle e tomada de decisão & Desenvolvido & \\
\hline
\end{tabular}

Fonte: Elaborado pelos autores (2018).

$\mathrm{O}$ aumento da eficiência técnica por acesso a insumos, tecnologia, treinamento e serviços, como resultado, é abordado por Abate, Francesconi e Getnet (2014). Em média, encontram uma diferença de 5\% na eficiência técnica entre membros da cooperativa e não membros. Verhofstadt e Maerstens (2014) relatam que os não membros gostariam de participar de cooperativas pelo aumento ao acesso a insumos, informação e crédito. Chagwiza, Muradian e Ruben (2016) indicam o aumento da adesão dos produtores rurais a inovações tecnológicas. Resultados estes que são associados à assistência técnica.

O resultado que tem maior ênfase aparece em 50\% das publicações, ou seja, seis das publicações trazem o aumento de renda dos cooperados a partir da participação. Ma e Abdulai (2017) relatam o aumento da renda em 1,83\%, Ma e Abdulai (2016) em 4,66\%, Mojo, Fischer e Degefa (2017) indicam que os cooperados teriam 26\% menos de renda se não participassem da cooperativa.

Verhofstadt e Maerstens (2014) relatam que a renda dos cooperados é $60 \%$ maior que a dos não-membros, e a renda dos cooperados da cooperativa de milho é $52 \%$ maior que a dos cooperados horticultores e o total de renda agrícola aumenta $25 \%$. Verhofstadt e Maerstens (2015) demonstram que a renda agrícola dos cooperados é maior, ou seja, de 580 dólares e de não-membros é de 260 dólares. Chagwiza, Muradian e Ruben (2016) não apresentam dados numéricos, apenas indicam o aumento da renda dos produtores de leite.

$\mathrm{O}$ aumento e a garantia de preço como resultado está presente nos três níveis de desenvolvimento. É um importante fator que leva os produtores rurais a se associarem nas 
cooperativas independente de sua situação econômica ou social. Para Mojo, Fischer e Degefa (2017,) quando as cooperativas começam a comprar café, todos os comerciantes de café elevam os preços para um nível igual ou, às vezes, melhor para vencer a concorrência emergente, o que beneficia todos os produtores.

Ma e Abdulai (2017) mostram que a participação cooperativa aumenta o preço da maçã em 8,82\% e Alho (2015) salienta que as cooperativas fornecem um preço competitivo ao produtor, sendo avaliado mais alto por $36 \%$ dos membros das cooperativas de suprimentos, e de $25 \%$ na amostra de cooperativas de comercialização.

A produtividade é resultado oriundo dos países em desenvolvimento. Kumar et al. (2018) apontam que a participação de uma sociedade cooperativa de laticínios tende a aumentar a produção de leite em $40 \%$ e Ma e Abdulai (2016) indicam o aumento nos rendimentos de produtividade de maçãs em $5,36 \%$. O retorno de investimento é designado como um benefício para os produtores rurais associados em cooperativa. Para Ma e Abdulai (2016), há o aumento do retorno em 6,06\% e Ma e Abdulai (2017), em 14\% no retorno das maçãs e Kumar et al. (2018), em 38 \% de aumento no retorno do leite.

A integração de mercado é evidenciada por um país desenvolvido e um subdesenvolvido. Alho (2015) retrata ser a cooperativa um canal de mercado estável com benefício significativo para $89 \%$ dos cooperados de laticínios e $83 \%$ dos cooperados de carne. Chagwiza, Muradian e Ruben (2016), na mesma perspectiva, demonstram a integração a um mercado confiável como um benefício aos produtores. Este resultado indica uma indiferença em relação ao nível de desenvolvimento do país.

O bem-estar dos produtores rurais é expresso por Ahmed e Mesfin (2017). Medindo as despesas de consumo por adultos equivalentes por ano, incluindo alimentos, roupas, moradia, educação e assistência médica, descobriu-se que a participação na cooperativa tem um consumo $26,5 \%$ maior por adultos equivalentes do que os não membros, revelando o aumento do bem-estar dos produtores rurais.

A redução da pobreza pela participação cooperativa é analisada por Verhofstadt e Maerstens (2015), os quais apontam a pobreza de forma generalizada na área de pesquisa, onde $34 \%$ dos domicílios dos cooperados são pobres e a incidência da pobreza é significativamente maior $(54 \%)$ entre os domicílios não-membros. A participação cooperativa reduz a probabilidade de ser pobre em $10 \%$ a $14 \%$ e tem um efeito médio de redução da pobreza na participação da cooperativa de $6,8 \%$. 
O capital humano, social e ambiental são fatores expressos por Mojo, Fischer e Degefa (2015). Para os produtores de café, há um impacto significativo das cooperativas no capital social dos membros, incluindo confiança, comprometimento e satisfação. No capital humano, há sessões de treinamento recebidas e experiências adquiridas. Já o desempenho ambiental dos produtores está negativamente associado com a adesão das cooperativas, devido à intensificação da produção do café provocando o desmatamento.

Segundo Ma, Abdulai e Goetz (2018), os membros de cooperativas são mais propensos a utilizar a adubação orgânica, 30,7\%, do que fertilizantes químicos, e que agregados familiares maiores têm mais probabilidade em investir nos fertilizantes químicos devido ao acesso à irrigação.

A partir da análise dos resultados, pode-se afirmar que esse estudo respondeu à questão da pesquisa. Também, identificou, a partir da bibliografia estudada, que a participação de produtores rurais em cooperativas agropecuárias, nos países com diferentes níveis de desenvolvimento, possui semelhanças e diferenças, dado o nível de desenvolvimento do país.

Quanto aos resultados em que se destacam os países subdesenvolvidos, há a preocupação com os aspectos de renda, preço, bem-estar, eficiência técnica, redução de pobreza, desempenho de capitais humanos sociais e ambientais e a integração de mercado, que são perspectivas sociais e econômicas. Sugere-se que os países subdesenvolvidos apresentam situação de fragilidade e que necessitam da figura das cooperativas agropecuárias para se superar e enaltecer suas atividades.

Deste modo, estas situações corroboram com os dados que justificam tais características dos resultados ao se classificar os países por nível de desenvolvimento. Em Ruanda, estes dados refletem que o índice de pobreza geral diminuiu cerca de $27,8 \%$ de 2000 a 2013, a taxa de incidência da pobreza rural representa 48,7\% da população rural, incidência que decresceu nos últimos 5 anos, quando antes era mais de 60\%. O grau de concentração de renda medido pelo Coeficiente de Gini no país é de 50,4 e sugere que há desigualdade entre os rendimentos dos indivíduos (BANCO MUNDIAL, 2018; UNDP, 2018).

Na Etiópia, o índice de pobreza geral diminuiu cerca de $20 \%$ de 1995 a 2017. A taxa de incidência da pobreza rural representa 30,4\% da população, incidência que decresceu nos últimos quinze anos. O grau de concentração de renda medido pelo 
Coeficiente de Gini no país é de 39,1, sugerindo que existem desigualdades entre os rendimentos dos indivíduos (BANCO MUNDIAL, 2018; UNDP, 2018).

Os países em desenvolvimento evidenciam o seu crescimento a partir do desenvolvimento econômico. Desta maneira, os resultados dos estudos demonstram que os países estão preocupados com o seu avanço e buscam por desempenho produtivo, retorno de investimentos, preço e renda, que são fatores determinantes para esses países alavancarem suas perspectivas de crescimento.

Os dados a seguir explicam as características dos resultados de países em desenvolvimento. $\mathrm{Na}$ China, $42 \%$ da população se encontram na área rural, o índice de pobreza geral diminuiu cerca de $14,1 \%$ de 2010 a 2017, a taxa de incidência da pobreza rural representa 7,2\% da população rural, o grau de concentração de renda medido pelo Coeficiente de Gini no país é de 42,2. Sugere-se que existem desigualdades entre os rendimentos dos indivíduos. (BANCO MUNDIAL, 2018; UNDP, 2018).

$\mathrm{Na}$ Índia, o índice de pobreza geral diminuiu cerca de 23,4\% de 1993 a 2011. A taxa de incidência da pobreza rural representa $25,7 \%$ da população, incidência que decresceu nos últimos dezoito anos de análise. O grau de concentração de renda medido pelo Coeficiente de Gini no país é de 35,1 sugerindo que existem desigualdades entre os rendimentos dos indivíduos (BANCO MUNDIAL, 2018; UNDP, 2018). Deste modo, salienta-se que o Coeficiente de Gini nos países em desenvolvimento é menor do que nos subdesenvolvidos, demonstrando que os primeiros têm menores desigualdades de rendimentos, quando comparados aos subdesenvolvidos.

Os países desenvolvidos se destacam em menos resultados e demonstram estar menos preocupados com aspectos considerados relevantes pelos países subdesenvolvidos e em desenvolvimento, pois os países desenvolvidos são avançados economicamente e socialmente garantindo aos seus produtores os aspectos fragilizados nos demais países. A sua preocupação principal é assegurar o preço, a integração de mercado e o controle na tomada de decisão. Na Finlândia, o grau de concentração de renda medido pelo Coeficiente de Gini é de 27,1, o que sugere a existência de desigualdade entre os rendimentos dos indivíduos, porém menor em relação aos demais países dos estudos (BANCO MUNDIAL, 2018; UNDP, 2018).

Ainda, pode-se notar que o preço de venda e a renda, que é gerada pela venda dos produtos comercializados com as cooperativas agropecuárias, são os principais resultados 
dos estudos em relação ao número de publicações e a presença nos três níveis de desenvolvimento. Os dados do Banco Mundial e do Programa de Desenvolvimento das Nações Unidas constatam a importância e as diferenciações dos resultados entre os níveis de desenvolvimento, dadas as suas características.

O índice de pobreza rural da população é maior entre os países subdesenvolvidos, o que indica, nos resultados dos estudos, a busca de melhorias sociais e econômicas, pois a população é mais pobre e a agricultura é sua fonte de subsistência. A renda proveniente da venda dos produtos agrícolas à cooperativa com determinado preço, como principal resultado, pode-se justificar ao analisar o Coeficiente de Gini dos países. Os resultados do coeficiente demonstram a importância da renda para a população dos países subdesenvolvidos e em desenvolvimento de modo a garantir o seu crescimento econômico.

O Índice de Desenvolvimento Humano (IDH) é determinante como indicador para justificar tais resultados vistos na revisão sistemática da literatura. Isso porque engloba três importantes dimensões utilizadas para classificar os países dos estudos por nível de desenvolvimento. O IDH indica as capacidades e as oportunidades para os indivíduos serem aquilo que desejam ser, e é medido por meio de três dimensões básicas: renda, educação e saúde. É revelado que o país com maior IDH é a Finlândia 0,920, seguida da China 0,752, Índia 0,640, Ruanda 0,524 e Etiópia 0,463 (UNDP, 2018).

Portanto, toda a interface desses resultados da amostra de estudos não deixa de expressar, independentemente do nível de desenvolvimento do país e suas diferenciações, o principal lema que influencia as cooperativas agropecuárias em todo o mundo. Segundo a International Co-operative Alliance (2018), as cooperativas têm o intuito de ser uma “associação autônoma de pessoas unidas voluntariamente para satisfazer suas necessidades e aspirações econômicas, sociais e culturais comuns por meio de uma empresa de propriedade conjunta e democraticamente controlada".

\section{CONSIDERAÇÕES FINAIS}

O presente estudo se propôs a investigar os condicionantes da participação de produtores rurais nas cooperativas agropecuárias em países com diferentes níveis de desenvolvimento enfatizando suas semelhanças e diferenças. Utilizou-se uma revisão sistemática da literatura, através de parâmetros estabelecidos nos procedimentos 
metodológicos. Chegou-se a uma amostra de doze artigos que tratam de países com diferentes níveis de desenvolvimento.

De modo geral, as cooperativas agropecuárias, baseadas principalmente nos princípios do cooperativismo, são essenciais para assegurar os pequenos produtores de tantas fragilidades sociais e econômicas nas áreas rurais. Assim, o importante papel destas organizações é indicado. A participação de produtores rurais nas cooperativas agropecuárias é reforçada por características positivas e semelhantes ao que se refere a benefícios e vantagens, como o aumento da renda, garantia de integração ao mercado e a eficiência técnica ou intensificação da agricultura que garante a produtividade dos locais em que foram realizados os estudos.

De acordo com o nível de desenvolvimento dos países, permitiu-se realizar o panorama das localidades, o qual influenciou na análise das diferenças entre os resultados em relação ao nível de desenvolvimento dos países e suas características que possam intervir nas evidências da participação de produtores rurais em cooperativas agropecuárias. Como visto, em virtude de diferenças, cada cooperativa agropecuária possui uma especialidade, podendo ser café, milho, leite ou carnes, que se difere nos países desde a produção desses alimentos até comercialização para as cooperativas.

Os países subdesenvolvidos são afetados por conflitos e tendências de baixo crescimento a longo prazo e as cooperativas, neste contexto, necessitam de incentivos para se fortalecer. Já nos países em desenvolvimento, apresentam-se alguns obstáculos em relação a práticas e resultados de produção, sendo que o governo nesses países tem papel fundamental para garantir o aperfeiçoamento das cooperativas. Os países desenvolvidos possuem atividades econômicas importantes na área agrícola que fomentam o crescimento social e econômico dos mesmos. Além das atividades básicas da agricultura, os produtores se utilizam de incrementos através do perfil geográfico das regiões para alavancar ainda mais a renda rural.

Portanto, pode-se analisar que cada país pertencente ao seu nível de desenvolvimento, possui características próprias, no que diz respeito às cooperativas agropecuárias e seus produtos comercializados. Essas se adequam às condições econômicas, sociais e demográficas do local, evidenciadas pelo IDH e o Coeficiente de Gini. 
Os principais limitadores do estudo foram a busca em apenas uma base de dados, restringindo maiores resultados, e as delimitações de anos usadas para filtrar os periódicos. Desta maneira, a utilização de outras bases e de um menor rigor nos filtros escolhidos podem aumentar a amostra de artigos e abranger também outros países para análise. A utilização da técnica de metanálise e ampliação da amostra de artigos para diversos outros países estão como sugestão para trabalhos futuros.

\section{REFERÊNCIAS}

ABATE, G. T.; FRANCESCONI, G. N.; GETNET, K. Impact of agricultural cooperatives on smallholders technical efficiency: empirical evidence from Ethiopia. Annals of Public and Cooperative Economics, v.85, n.2, p.257-286, 2014.

AHMED, M. H.; MESFIN, H. M. The impact of agricultural cooperatives membership on the wellbeing of smallholfer farmers: empirical evidence from eastern Ethiopia. Agricultural Food Economics, v.5, n.6, p.1-20, 2017.

ALENCAR, E. S.; ALMOULOUD, S. A. A metodologia de pesquisa: metassíntese qualitativa. Revista Reflexão e Ação, Santa Cruz do Sul, v.25, n.3, p.204-220, 2017.

ALHO, E. Farmers self-reported value of cooperative membership: evidence from heterogeneous business and organization structures. Agricultural and Food Economics, v.3, n.23, p.1-22, 2015.

BANCO MUNDIAL. World Bank Open Data: free and open Access to global development data. 2018, The World Bank, Washington, D.C, EUA. Disponível em: <https://data.worldbank.org/>. Acesso em: 25 nov. 2018.

BRESSER-PEREIRA, L. C. Desenvolvimento, progresso, e crescimento econômico. Lua Nova, São Paulo, 2014, 93: p.33-60. Disponível em:

<http://www.scielo.br/pdf/ln/n93/03.pdf $>$. Acesso em: 09 dez. 2018.

CHADDAD, F. R.; COOK, M. L. Understanding new cooperative models: an ownershipcontrol rights typology. Review of Agricultural Economics, Oxford, United Kingdom, v.26, n.3, p.348-360, Autumn 2004.

CHAGWIZA, C.; MURADIAN, R.; RUBEN, R. Cooperative membership and performance among smallholders in Ethiopia. Food Policy, v.59, n.1, p.165-173, 2016.

DEKA, R. et al. The white revolution in India: the end or a new beginning? Poster prepared for the Agri4D 2015 conference, Uppsala, Sweden, p. 23-24. Nairobi, Kenya: ILRI. 2015. Disponível em: <https://cgspace.cgiar.org/handle/10568/71012>. Acesso em: 25 nov. 2018. 
FAO. Food and Agriculture Organization of the United Nation. Agricultural cooperatives: paving the way for food security and rural development, 2012 . Disponível em: <http://www.fao.org/3/ap088e/ap088e00.pdf>. Acesso em: 19 nov. 2018.

Cooperatives and producer organizations. 2018a, Disponível em: <http://www.fao.org/partnerships/cooperatives/en/>. Acesso em: 19 nov. 2018.

(2018b). Country Profiles. Disponível em: <http://www.fao.org/countryprofiles/en/>. Acesso em: 19 nov. 2018.

FRANCESCONI, G. N. A new generation of cooperatives for Africa. CIAT Policy Brief. $2017 . \quad$ Disponível em: <https://www.researchgate.net/publication/320709640_A_New_Generation_of_Cooperativ es_for_Africa>. Acesso em: 25 nov. 2018.

ICA. International Co-operative Alliance. Cooperatives, 2018. Disponível em: <https://www.ica.coop/en>. Acesso em: 02 dec. 2018.

KUMAR, A. et al. Does cooperative membership improve household welfare? evidence from a panel data analysis of smallholder dairy farmers in Bihar, Índia. Food Policy, v.75, p.24-36. 2018.

LAUSCHNER, R. Agribusiness, cooperativa e produtor rural. 2. ed. São Leopoldo-RS: Editora Unisinos, 1993.

LIMA, P. O. et al. Perfil dos produtores rurais do município de Quixeramobim no stado do Ceará. Revista Caatinga, Mossoró, v.22, n.4, p.255-259. 2009. Disponível em: <https://www.redalyc.org/pdf/2371/237117843037.pdf>. Acesso em: 30 nov. 2018.

LOPES, A. L. M.; FRACOLLI, L. A. Revisão sistemática de literatura e metassíntese qualitativa: considerações sobre sua aplicação na pesquisa em enfermagem. Texto Contexto Enfermagem, Florianópolis, v.17, n.4, p.771-778, dez. 2008.

MA, W.; ABDULAI, A. Does cooperative membership improve household welfare? evidence from apple farmers in China. Food Policy, v.58, n.1, p.94-102. 2016.

The economic impacts of agricultural cooperatives on smallholder farmers in rural China. Agribusiness, v.33, n.4, p.537-551. 2017.

MA, W.; ABDULAI, A.; GOETZ, R. Agricultural cooperatives and investment in organic soil amendments and chemical fertilizer in China. American Journal of Agricultural Economics, v.100, n.2, p.502-520, 2018.

MOJO, D.; FISCHER, C.; DEGEFA, T. Social and environmental impacts of agricultural cooperatives: evidence from Ethiopia. International Journal of Sustainable Development and World Ecology, v.22, n.5, p.388-400, 2015. 
. The determinants and economic impacts of membership in coffe farmer cooperatives: recent evidence from rural Ethiopia. Journal of Rural Studies, v.50, n.1, p.84-94, 2017.

NIELSEN, L. How to classify countries based on their level of development. Soc. Indic. Res. 114:1087-1107. 2013. Disponível em: <https://link.springer.com/content/pdf/10.1007/s11205-012-0191-9.pdf $>$. Acesso em: 30 nov. 2018.

OIT. Organização Internacional do Trabalho (2002). Recomendação $\mathbf{n}^{\mathbf{0}} 193$ sobre a promoção de cooperativas de 03 de junho de 2002. Disponível em: <http://www.sescoopsp.org.br/sms/files/file/Recomenda\%C3\%83\%C2\%A7\%C3\%83\%C2 \%A3o\%20193\%20OIT.pdf>. Acesso em: 20 mar. 2019.

PINHO, D. B. O pensamento cooperativista e o cooperativismo brasileiro. São Paulo: Saraiva, 1982.

SALES, J. E. Cooperativismo: origens e evolução. Revista Brasileira de Gestão e Engenharia, Centro de Ensino Superior de São Gotardo, n.1. p. 23-24, 2010. Disponível em: $\quad\langle$ https://periodicos.cesg.edu.br/index.php/gestaoeengenharia/article/view/30/23 > . Acesso em: 17 set. 2019.

SAMPAIO, R. F.; MANCINI, M. C. Estudos de revisão sistemática: um guia para síntese criteriosa da evidência científica. Revista Brasileira de Fisioterapia, São Carlos, v.11, n.1, p.83-89. 2007.

SEN, A. K. Desenvolvimento como liberdade. São Paulo: Companhia das Letras. 1999. Disponível em:

$<$ https://edisciplinas.usp.br/pluginfile.php/19539/mod_resource/content/2/CHY\%20\%20Sen\%20-\%20Aula\%208.pdf>. Acesso em: 09 dez. 2018.

TEIXEIRA, F. R. et al. Evolução histórica do cooperativismo no setor agropecuário. Id on Line: Revista Multidisciplinar e de Psicologia. v.11, n.39. 2017. Edição eletrônica Disponível em: <https://idonline.emnuvens.com.br/id/article/view/939/1409>. Acesso em: 19 nov. 2018.

UNDP. United Nations Development Programme. Human development indices and indicators 2018 statistical update. 2018. Disponível em: <http://hdr.undp.org/en/2018update>. Acesso em: 02 dec. 2018.

USDA. India dairy and products annual 2017, Browse Data \& Analysis, United States Department of Agriculture Foreign Agricultural Service. 2017b. Disponível em: <https://apps.fas.usda.gov/newgainapi/api/report/downloadreportbyfilename?filename=Dai ry\%20and\%20Products\%20Annual_New\%20Delhi_India_10-13-2017.pdf>. Acesso em: 25 nov. 2018. 
VERHOFSTADT, E.; MAERTENS, M. Smallholder cooperatives and agricultural performance in Rwanda: do organizational diferences matter? Agricultural Economics (United Kingdom), v.45, n.1, p.39-52, 2014.

Can agricultural cooperatives reduce poverty? heterogenous impact of cooperative membership on farmers welfare in Rwanda. Applied Economic Perspectives anda Policy, v.37, n.1, p.86-106, 2015. 